\title{
How achievable are free sugar recommendations in healthy dietary patterns for 1-5 year olds in Ireland?
}

\author{
$\underline{\text { S.F. Quinn }}^{1}$, R.J. Creane ${ }^{1}$, C.B. O’Donovan ${ }^{1}$, S. O’Mahony ${ }^{1,2}$, N.A. Collins ${ }^{1}$, O.C. Lyons ${ }^{1,3}$ and \\ M.A.T. Flynn ${ }^{1,3}$ \\ ${ }^{1}$ Food Safety Authority of Ireland, Dublin 1, Ireland, \\ ${ }^{2}$ University College Dublin, Dublin 4, Ireland and \\ ${ }^{3}$ Nutrition Innovation Centre for Food and Health, Ulster University, Coleraine, United Kingdom
}

The World Health Organisation (WHO) strongly recommends reducing free sugar intake to $<10 \%$ energy, and suggests a further conditional reduction to $<5 \%$ energy. This study aims to assess how achievable these free sugar intake recommendations are in healthy diets of 1-5 year olds in Ireland and to identify the main food contributors.

Using minimal added sugar, fat and no added salt, four-day food intake patterns were developed for $1-5$ year old children ( $n 30$ girls; $n 30$ boys) representing different percentile levels on the WHO growth charts. These food intake patterns were based on foods commonly consumed in the Irish National Pre-school Nutrition Survey and adjusted to meet energy, macronutrient and micronutrient requirements. The free sugar content of each food used was identified according to the WHO definition and estimated using food composition tables. Free sugar content of the four-day patterns was assessed against the two WHO recommendations. Subjects were compared depending on recommendations achieved. Where necessary, nutrients were estimated per 500kcal to control for energy.

Food intake patterns of all subjects ( $n 60)$ achieved the WHO recommendation of $<10 \%$ energy, with $50 \%(n 30)$ meeting the further recommendation of $<5 \%$ energy. Subjects with free sugar intakes $<5 \%$ energy compared with those not meeting this recommendation, were younger ( 2 years vs. 3.5 years, $\mathrm{p}<0.001)$, had lower energy intakes $(946 \mathrm{kcal} v s .1263 .8 \mathrm{kcal}, \mathrm{p}<0.001)$, higher fat intakes (37\% vs. $29 \%, \mathrm{p}<0.001)$, higher saturated fat intakes $(18 \%$ vs. $13 \%, \mathrm{p}<0.001)$ and lower carbohydrate intakes $(46 \%$ vs. $53 \%, \mathrm{p}<$

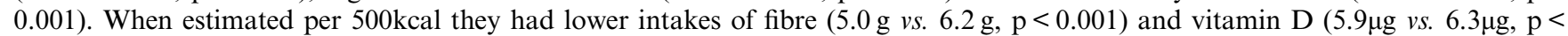
$0.001)$ and higher intakes of vitamin A $(177.3 \mu \mathrm{g} v s .108 .5 \mu \mathrm{g}, \mathrm{p}<0.001)$ and DHA and EPA $(0.3 \mathrm{~g} v s .0 .2 \mathrm{~g}, \mathrm{p}<0.001)$. The main foods contributing to free sugar intakes in these subjects were cereals (29\% of free sugar intake), yoghurts $(27 \%)$, breads $(5 \%)$ and ice cream $(4 \%)$. By comparison the main foods contributing to free sugar in subjects whose intakes exceeded $5 \%$ energy included yoghurts $(22 \%)$, stewed apples $(9 \%)$ and puddings $(6 \%)$.

This analysis demonstrates the WHO strong recommendation of $<10 \%$ energy for free sugar was easily achievable, but the further conditional recommendation $(<5 \%$ ) was more difficult. This was only achieved at younger ages and was associated with increased intakes of fat and saturated fat and lower intakes of fibre and vitamin D. The main food contributors to free sugar in healthy diets for 1-5 year olds are important sources of nutrients.

\section{Conflict of Interest}

There is no conflict of interest 